\title{
Simulación clínica en vuelo para el manejo del paciente crítico en aeroevacuación avanzada y táctica en Afganistán
}

\author{
Munayco Sánchez AJ. ${ }^{1}$, Tabla Hinojosa FB. ${ }^{2}$, García Noguera A. ${ }^{3}$ \\ Sanid. mil. 2013; 69 (3): 210-212; ISSN: 1887-8571
}

\begin{abstract}
RESUMEN
Los diferentes relevos de tripulaciones sanitarias en el puesto de MEDEVAC perteneciente al destacamento HELISAF en Herat requieren coordinación y trabajo en equipo en condiciones adversas. Por este motivo se decidió realizar adiestramiento mediante códigos mega con simuladores que permitieran poner en práctica técnicas de soporte vital avanzado con incidencias múltiples. Exponemos el escenario de trabajo, las características especiales de la simulación, el procedimiento empleado, las recomendaciones específicas asistenciales y la conclusión de la experiencia. El artículo desarrolla la experiencia obtenida al realizar prácticas de simulación clínica durante el vuelo de helicópteros HD-21 «Superpuma» en Afganistán.
\end{abstract}

Palabras clave: MEDEVAC, HELISAF, ROLE2E, Simulación.

Flight clinical simulation for critical care support in forward and tactical aeroevacuation in Afghanistan SUMMARY

Medical crews in MEDEVAC job description that belongs to HELISAF deployment requires coordination and teamwork in adverse conditions. For this reason, were performed clinical simulations to practice advance life support with some incidents. Theater system and special conditions in the simulations are described. As well as procedures and specific care recommendations. Finally conclusions are presented. The purpose of this report is to show the experience gained in performing clinical simulation in HD-21 «Superpuma» helicopter in Afghanistan.

Key words: MEDEVAC, HELISAF, ROLE2E, Simulation.

\section{INTRODUCCIÓN}

Es bien conocida la enorme utilidad de la simulación clínica ${ }^{1}$ para el aprendizaje, consolidación y mantenimiento de las habilidades asistenciales en el manejo del paciente crítico en sus diferentes niveles.

La asistencia a un paciente que requiere muchas técnicas complejas simultáneas y rápidas con decisiones asistenciales inmediatas, no se improvisa y requiere un entrenamiento continuado adecuado a la actividad a realizar en las condiciones más parecidas.

Desde el 2005 en Herat (Afganistán), se desplegó el destacamento HELISAF con personal sanitario compuesto por dos oficiales médicos de vuelo, dos oficiales enfermeros tripulantes SAR y dos MPTM de logística aérea, teniendo como principal misión la realización de aeroevacuaciones avanzadas y tácticas.

Constituye una asistencia al paciente crítico, generalmente más complicada que la realizada en el entorno civil.

Partimos del condicionante de hostilidad ${ }^{2,3}$ representado por encontrarnos en una zona de conflicto, con la necesidad de trabajar con un equipo personal de vuelo (constituido por chaleco

${ }^{1}$ Cap. Médico. Unidad Médica Aérea de Apoyo al Despliegue Madrid. España.

${ }^{2}$ Cap. Médico. Ala 23, Talavera la Real. Badajoz. España.

${ }^{3}$ Cap. Enfermero. Ala 78, Armilla. Granada. España.

Dirección para correspondencia: Armando José Munayco Sánchez. Base aérea de Torrejón. 28850, Torrejón de Ardoz. Email: delascuevs@hotmail.com

Recibido: 27 de noviembre de 2012

Aceptado: 20 de mayo de 2013 balístico, casco balístico, gafas de visión nocturna, arnés de seguridad, transmisiones, etc...), con un peso aproximado de 20 $\mathrm{kg}$, en donde se atiende a un politraumatizado por explosivo o por arma de fuego, en un entorno eminentemente aéreo que difiere del civil en el que predomina la asistencia sobre el terreno ${ }^{4}$.

Las condiciones de asistencia en vuelo son complicadas. Dentro de la cabina hay que soportar frecuentemente fuertes rachas de viento, temperaturas extremas, poca luminosidad en vuelos nocturnos, aceleraciones, vibraciones, cambios de presión, etc. Es un espacio reducido que precisa de tenerlo todo previsto para moverse lo menos posible por el área asistencial.

La mejor manera de entrenar adecuadamente, las habilidades aprendidas y la coordinación entre el equipo asistencial es a través de la simulación en el medio aéreo ${ }^{4}$.

\section{MATERIAL Y MÉTODOS}

Estudio retrospectivo realizado en un periodo de 80 días comprendido entre abril y junio de 2012, por dos equipos (A y B) de aeroevacuación médica (MEDEVAC), compuesto cada uno de ellos por un oficial Médico, un oficial Enfermero y un MPTM.

Con objeto de conseguir un nivel básico inicial de coordinación entre los miembros del equipo sanitario, se realizaron 4 simulaciones de preparación en las instalaciones sanitarias del Role 2 de Herat (Afganistán) con un simulador de Soporte Vital Avanzado $\left(\right.$ Laerdal $^{\circledR}$ ) que permite RCP avanzada con manejo avanzado de la vía aérea, además de canalización de vía venosa. 


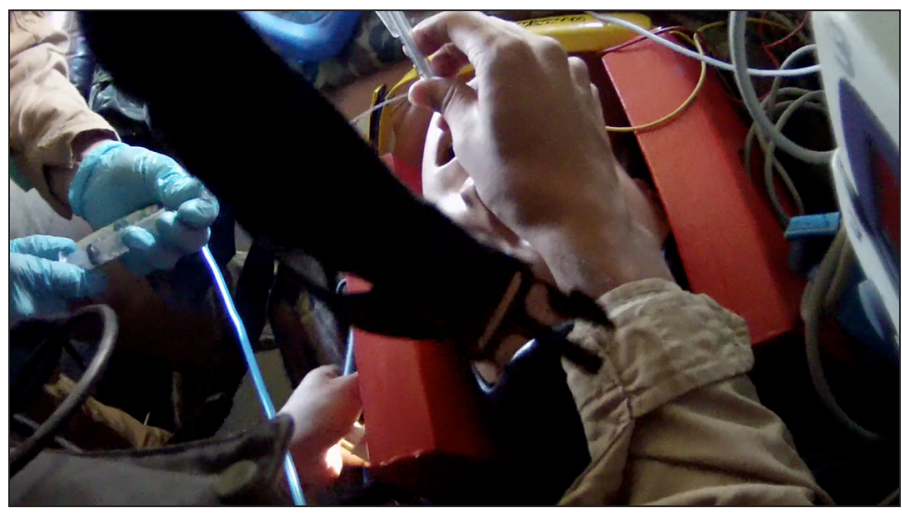

Figura 1. Realización del primer caso de simulación. Fotografía de los autores.

Posteriormente durante vuelos de instrucción para las tripulaciones se realizaron 5 simulaciones clínicas (Figura 1 y 2 ).

Se desarrollaron 5 casos clínicos (Tabla 1) tratando de reproducir diversas contingencias que pueden aparecer por evolución del herido o por fallo en los dispositivos de electromedicina, valorando la repercusión del vuelo sobre los procedimientos realizados en los heridos. Los tres componentes del equipo sanitario fueron rotando su papel asistencial.

Para la simulación clínica en vuelo se empleó el mismo simulador de Soporte Vital Avanzado (Laerdal ${ }^{\circledR}$ ) con material fungible adecuado a los procedimientos que había sobrepasado la fecha de caducidad (con el deseo de no interferir en los niveles logísticos de los recursos clase VIII) y con dispositivos de electromedicina.

Se analizaron los siguientes parámetros: valoración primaria, intubación orotraqueal, dispositivos supraglóticos, manejo avanzado de la vía aérea y otros procedimientos (colocación de torniquete, empleo de agentes hemostáticos, punción torácica, drenaje torácico, parche torácico, vía intravenosa, vía intraósea). Así mismo se estudiaron las condiciones generales del vuelo (horario diurno/nocturno, altitud máxima media, presión mínima media y temperatura mínima media)

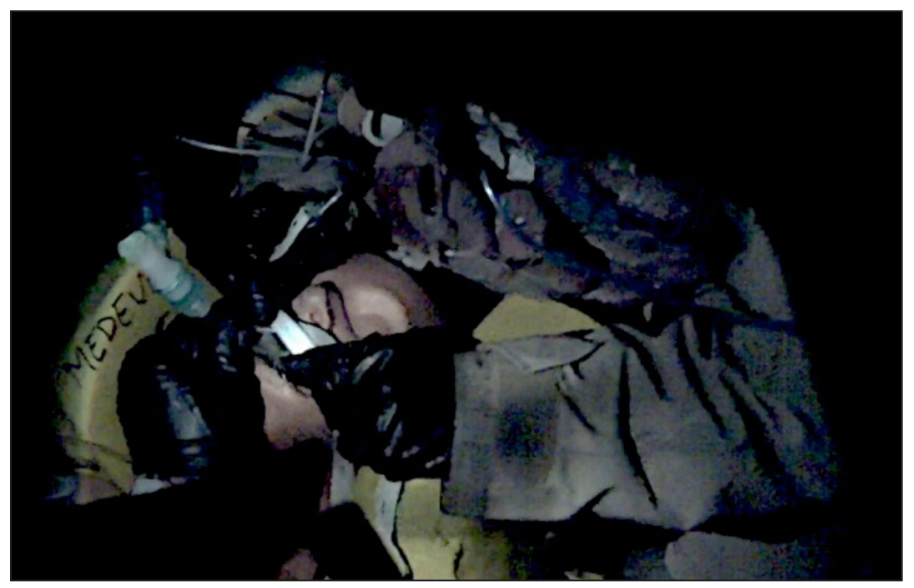

Figura 2. Realización del tercer caso de simulación durante un vuelo nocturno. Fotografía de los autores.

\section{RESULTADOS}

Durante el tiempo del estudio se realizaron 5 vuelos. El equipo asistencial A realizó tres vuelos de simulación clínica, mientras que el equipo asistencial B realizó dos vuelos. El equipo A simuló los casos número 1,2 y 3 , mientras que el equipo $\mathrm{B}$ realizó los supuestos 4 y 5.

Los principales parámetros aeronáuticos fueron 4 vuelos diurnos y 1 nocturno, altitud máxima media de $1365 \mathrm{~m}$, presión mínima media de $861,5 \mathrm{mmHg}$ y temperatura mínima media de $19^{\circ} \mathrm{C}$.(Figura 3)

Los resultados de las técnicas y procedimientos realizados fueron los siguientes:

a. Valoración primaria: El número de integrantes del equipo sanitario es el adecuado. Es necesario el reparto de las tareas del CABCDE de forma previa al comienzo del vuelo. Es recomendable minimizar los movimientos del personal asistencial en la cabina y restringir las comu-

Tabla 1. Descripción de los casos clínicos simulados.

\begin{tabular}{|c|c|c|c|c|c|}
\hline Caso & Mecanismo & Lesiones & Procedimiento & Complicaciones & Procedimiento \\
\hline 1 & Blast IED & $\begin{array}{l}\text { Amputación MID, Shock } \\
\text { hemorrágico }\end{array}$ & $\begin{array}{l}\text { Hemostasia. } \\
\text { Manejo avanzado vía aérea. } \\
\text { Fluidoterapia y farmacoterapia }\end{array}$ & $\begin{array}{l}\text { Neumotórax a tensión. Fugas } \\
\text { en sistema de Ventilación }\end{array}$ & $\begin{array}{l}\text { Descompresión. } \\
\text { Resolución de contingencia }\end{array}$ \\
\hline 2 & Blast IED & $\begin{array}{l}\text { Hemorragia exanguinante } \\
\text { MSD, quemaduras faciales. } \\
\text { Simulador en camilla } \\
\text { inferior }\end{array}$ & $\begin{array}{c}\text { Hemostasia. } \\
\text { Manejo avanzado vía aérea. } \\
\text { Fluidoterapia y farmacoterapia. } \\
\text { Procedimiento en la camilla } \\
\text { inferior }\end{array}$ & $\begin{array}{l}\text { PCR por disociación } \\
\text { electromecánica en Shock } \\
\text { hemorrágico. }\end{array}$ & RCP avanzada y resucitación \\
\hline 3 & $\begin{array}{l}\text { Accidente de tráfico } \\
\text { tras ataque IED. } \\
\text { Vuelo nocturno }\end{array}$ & $\begin{array}{c}\text { TCE moderado. Trauma } \\
\text { torácico con neumotórax a } \\
\text { tensión }\end{array}$ & Descompresión & TCE severo. Herniación. & $\begin{array}{c}\text { Manejo avanzado vía aérea, } \\
\text { fluidoterapia y farmacoterapia. } \\
\text { Valoración secundaria }\end{array}$ \\
\hline 4 & $\begin{array}{l}\text { Impacto proyectil } \\
\text { durante TIC }\end{array}$ & $\begin{array}{l}\text { Fractura por estallido de } \\
\text { cabeza de húmero izq. } \\
\text { y neumotórax abierto } \\
\text { colateral. }\end{array}$ & $\begin{array}{l}\text { Colocación mecanismo valvular } \\
\text { torácico . Inmovilización MSI. } \\
\text { Acceso intraóseo MII y acceso } \\
\text { venoso MSD. Fluidoterapia y } \\
\text { farmacoterapia. }\end{array}$ & Paciente chocado. & $\begin{array}{c}\text { Revisión fluidoterapia y } \\
\text { oxigenoterapia. } \\
\text { Descenso al máximo de línea } \\
\text { de vuelo. }\end{array}$ \\
\hline 5 & Blast IED & $\begin{array}{l}\text { Amputación traumática } \\
\text { MID, TCE y Trauma facial }\end{array}$ & $\begin{array}{c}\text { Torniquete, Manejo avanzado vía } \\
\text { aérea }\end{array}$ & $\begin{array}{l}\text { Resangrado, contingencias } \\
\text { ventilador }\end{array}$ & $\begin{array}{c}\text { Revisión torniquete, } \\
\text { fluidoterpia y resolución } \\
\text { contingencias }\end{array}$ \\
\hline
\end{tabular}


nicaciones durante la misión. Es adecuado que las diluciones de los fármacos más frecuentemente empleados se realicen antes del inicio del vuelo y que cada miembro lleve el material sanitario básico que va a emplear.

b. Intubación orotraqueal: Se objetivó que la posición ideal de la baja es con la cabeza del paciente hacia proa manteniendo las puertas cerradas, sin embargo también se comprobó el éxito en la intubación si la colocación de la baja era al contrario (cabeza hacia cola) o si el herido se encontraba en la camilla inferior (tanto con cabeza hacia proa como hacia cola) siendo necesario en estos tres últimos casos una adecuada redistribución del equipo asistencial. Fue recomendable el reparto previo de tareas en el manejo de $\mathrm{O}_{2}$, del ventilador y de la baja para mejorar la eficacia durante el procedimiento de la intubación. Se mostró la necesidad de estar adiestrado en la secuencia de intubación rápida adaptada al vuelo. Así mismo, el dispositivo Airtrack fue considerado como un adecuado método alternativo para el aislamiento de la vía aérea.

c. Dispositivos supraglóticos: Fueron evaluados la mascarilla laríngea, el tubo laríngeo y la mascarilla fastrack. Se objetivó la necesidad de implementar la sujeción de los tres dispositivos a la cara debido a la presencia de vibraciones y a las características propias del vuelo táctico. Los parámetros ventilatorios recogidos en la curva de presión indicaron una función ventilatoria disminuida.

d. Otros procedimientos (colocación de torniquete, empleo de agentes hemostáticos, punción torácica, drenaje torácico, parche torácico, vía intravenosa, vía intraósea), fueron realizados con éxito durante las simulaciones. Destacó la ventaja de portar dispositivos específicos en el equipo personal mejorando notablemente su accesibilidad.

\section{DISCUSIÓN}

Las exclusivas condiciones de vuelo del HD-21 en el Teatro de Operaciones obligan a realizar entrenamientos periódicos que garanticen la eficacia durante la asistencia al paciente crítico.

Los procedimientos que hemos valorado más adecuados y que deben ser reevaluados y actualizados son:

Establecimiento previo de la distribución del equipo asistencial en función de la colocación de los heridos.

Preparación previa al traslado del herido de las diluciones y fluidoterapia.

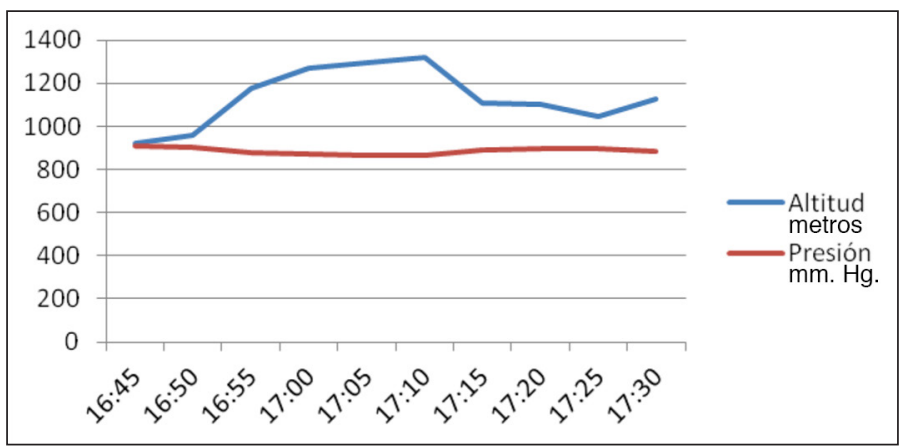

Figura 3. Perfil aeronáutico del vuelo número 1.
Dominio del manejo avanzado de la vía aérea con establecimiento de una secuencia rápida de intubación adaptada al vuelo (debe ser consensuada).

El Airtrack es buena alternativa.

Los dispositivos supraglóticos más adecuados son la máscara laríngea y el tubo laríngeo, sencillos de colocar, con pocas piezas aunque precisan mejorar los sistemas de fijación. Son dispositivos que hay que incluir en la secuencia rápida de intubación.

Manejo avanzado de la sedoanalgesia y ventilación mecánica.

Llevar en el equipo personal de vuelo dispositivos propios de asistencia inicial al trauma en combate (torniquetes, agentes hemostáticos, cánulas nasofaríngeas, aguja punción torácica, parches torácicos, dispositivos de vía intraósea).

Debemos valorar la creación de una doctrina completa en el ámbito de las Urgencias, Emergencias y Medicina Logísticooperativa en el contexto de un Diploma de Acreditación.

\section{CONCLUSIONES}

De nuestra experiencia asistencial y de las simulaciones en vuelo extraemos que la asistencia al paciente crítico en vuelo constituye un deporte de equipo que requiere la aptitud adecuada con un entrenamiento continuado y coordinado del equipo asistencial. Las tripulaciones sanitarias deben conocerse en estos escenarios para llegar a optimizar la asistencia, siendo eficaces y rápidos en la práctica clínica.

Las experiencias descritas en el presente informe nos demostraron claramente la enorme utilidad de la simulación, al realizarse en las condiciones exclusivas sólo posible en el teatro de operaciones, que además permitieron ensayar y mejorar los diferentes procedimientos asistenciales previamente aprendidos.

Los simulacros realizados mejoraron notablemente nuestra habilidad asistencial como Tripulación Sanitaria y como Equipo Avanzado de Trauma 5 .

Destaca la necesidad de realizar simulaciones estáticas y en vuelo como condición para considerar operativas las tripulaciones sanitarias en el teatro de operaciones.

De igual manera se nos plantea las enormes posibilidades en la mejora de nuestra práctica asistencial ante la posibilidad de realizar simulaciones clínicas regladas en vuelo en territorio nacional, reproduciendo en gran medida las condiciones descritas.

El paciente crítico nos exige máxima formación y eficacia en las situaciones descritas, la simulación en vuelo contribuye a ello, pero debemos incluirlo en el contexto de una formación reglada dentro de un cuerpo doctrinal como Diploma de Acreditación propio.

\section{BIBLIOGRAFÍA}

1. Foliente R. Combat engineers conduct air medical evacuation training. [Internet]. Diwaniya (Iraq): Defense video and imagery distribution System; 2009 (acceso 8 de febrero 2013). Disponible en: www.dvidshub.net/news/30953

2. Small Unit Operations in Afghanistan. Tactics, techniques and procedures. Handbook No09-37: US Unclassified; 2009.

3. Tactical Combat Casualty care. Tactics, techniques and procedures. Handbook N $N^{\circ}$ 10-44: US Unclassified; 2010.

4. Ostlund W.B. Tactical Lider lessons learned in Afghanistan Operation enduring Freedom VIII. Mil Rev (Fort Leavenworth). Julio-agosto 2009; 2-9. 